\title{
Coreshine in L1506C - Evidence for a primitive big-grain component or indication for a turbulent core history?
}

\author{
J. Steinacker ${ }^{1,2}$, C. W. Ormel ${ }^{3}$, M. Andersen ${ }^{1}$, and A. Bacmann ${ }^{1}$
}

\author{
1 UJF-Grenoble 1/CNRS-INSU, Institut de Planétologie et d'Astrophysique de Grenoble (IPAG) UMR 5274, 38041 Grenoble, France \\ e-mail: stein@mpia.de, morten.andersen@obs.ujf-grenoble.fr, aurore.bacmann@ujf-grenoble.fr \\ 2 Max-Planck-Institut für Astronomie, Königstuhl 17, 69117 Heidelberg, Germany \\ 3 Astronomy Department, University of California, Berkeley, CA 94720, USA \\ e-mail: ormel@astro.berkeley.edu
}

Received 21 June 2013 / Accepted 13 March 2014

\begin{abstract}
Context. With the initial steps of the star formation process in the densest part of the interstellar medium (ISM) still under debate, much attention is paid to the formation and evolution of pre-stellar cores. The recently discovered coreshine effect can aid in exploring the core properties and in probing the large grain population of the ISM.

Aims. We discuss the implications of the coreshine detected from the molecular cloud core L1506C in the Taurus filament for the history of the core and the existence of a primitive ISM component of large grains becoming visible in cores.

Methods. The coreshine surface brightness of L1506C is determined from Spitzer IRAC images at $3.6 \mu \mathrm{m}$. We perform grain growth calculations to estimate the grain size distribution in model cores similar in gas density, radius, and turbulent velocity to L1506C. Scattered light intensities at $3.6 \mu \mathrm{m}$ are calculated for a variety of MRN and grain growth distributions using the DIRBE $3.5 \mu \mathrm{m}$ all-sky map as external interstellar radiation field, and are compared to the observed coreshine surface brightness.

Results. For a core with the overall physical properties of L1506C, no detectable coreshine is predicted with a size distribution following the shape and size limits of an MRN distribution. Extending the distribution to grain radii of about $0.65 \mu \mathrm{m}$ allows to reproduce the observed surface brightness level in scattered light. Assuming the properties of L1506C to be preserved, models for the growth of grains in cores do not yield sufficient scattered light to account for the coreshine within the lifetime of the Taurus complex. Only increasing the core density and the turbulence amplifies the scattered light intensity to a level consistent with the observed coreshine brightness.

Conclusions. The coreshine observed from L1506C requires the presence of grains with sizes exceeding the common MRN distribution. The grains could be part of primitive omni-present large grain population becoming visible in the densest part of the ISM, could grow under the turbulent dense conditions of former cores, or in L1506C itself. In the later case, L1506C must have passed through a period of larger density and stronger turbulence. This would be consistent with the surprisingly strong depletion usually attributed to high column densities, and with the large-scale outward motion of the core envelope observed today.
\end{abstract}

Key words. dust, extinction - ISM: clouds - infrared: ISM - ISM: individual objects: L1506 - scattering

\section{Introduction}

Star formation research has identified the densest parts of molecular clouds as the sites where stars form. But the entire process from forming the molecular cloud, through the formation of the cloud cores, its evolution while having no central object, to the final collapse is not understood (Bergin \& Tafalla 2007). Physically, we are facing a coupled problem of gas dynamics including turbulent motions, gravitation, and magnetic fields where cooling and heating of the gas and dust are influenced by the chemical processes, grain processing, and the external illumination.

The theory of gravo-turbulent star formation predicts that molecular cloud cores form at the stagnation points of the complex turbulent flow pattern (Mac Low \& Klessen 2004). But since supersonic turbulence does not create hydrostatic equilibrium configurations, the density structures are transient and dynamically evolving, as the different contributions to virial equilibrium do not balance (Vázquez-Semadeni et al. 2003). Therefore, a statistical approach in collecting core properties has been a main approach to study their evolution. For individual cores, their history has been accessed by studying their chemical state (Pagani et al. 2013).

The filamentary Taurus star formation region (see, e.g., Qian et al. 2012) is an ideal site to test these theories and to determine the properties of cores due to its proximity and coverage of all low-mass star formation stages. In a recent analysis of a Taurus filament fragment L1506C based on dust emission, and line emission of $\mathrm{C}^{18} \mathrm{O}, \mathrm{N}_{2} \mathrm{H}^{+},{ }^{13} \mathrm{CO}$, and $\mathrm{C}^{17} \mathrm{O}$, Pagani et al. (2010a, Pea10 hereafter) show that the fragment combines interesting and surprising properties. Interpreted as a core-envelope system, the core located within $3 \times 10^{4}$ au shows low densities $n\left(\mathrm{H}_{2}\right)<5 \times 10^{10} \mathrm{~m}^{-3}$, but high depletion $>30$ of $\mathrm{C}^{18} \mathrm{O}$ and low temperatures $8-10 \mathrm{~K}$ depending on the tracer. Kinematic information was derived from line emission measured along a cut through the filament. The line-of-sight integrated emission was modeled with a 1D line transfer code. The authors argued that the line profiles show evidence for contraction of the core plus rotation with an infall speed of about $100 \mathrm{~m} / \mathrm{s}$. In turn, the velocity gradient of the envelope visible in the ${ }^{13} \mathrm{CO}$ line was found to be opposite in direction to that of the core as traced by the $\mathrm{C}^{18} \mathrm{O}$ line. Moreover, the core showed extremely low turbulence 
$v_{\text {turb }}(F W H M)<68 \mathrm{~m} / \mathrm{s}$. Based on the contraction of the core and its low density, the authors concluded that the core is about to become a pre-stellar core, but has not yet reached the density criterion suggested by Keto \& Caselli (2008) for a pre-stellar core.

An important piece of evidence was added to this picture by Pagani et al. (2010b). They reported on the detection of coreshine for about half of 110 investigated cores, among them L1506C. According to the analysis presented in Steinacker et al. (2010) for the core L183, the interstellar radiation can be scattered at wavelengths around $4 \mu \mathrm{m}$ when the core gas hosts dust grains which are about a factor of 4 larger than the maximal silicate grain size of about $0.25 \mu \mathrm{m}$ advocated by the Mathis, Rumpl, and Nordsieck (MRN) model.

Investigating Herschel observations of the entire filament harboring L1506C, Ysard et al. (2013) found that the grain opacity has to increase across the filament to fit simultaneously the near-IR extinction and FIR Herschel emission profiles. They interpreted this change to be a consequence of the coagulation of dust grains to form fluffy aggregates with the grain average size being increased by a factor of 5 .

In this paper, we analyze the coreshine seen towards L1506C and discuss its implication for the underlying grain size distribution and the history of the core. In Sect. 2, we derive the range of coreshine surface brightness being consistent with the IRAC $3.6 \mu \mathrm{m}$ data of L1506C. For a model core, we calculate the scattered light intensity for MRN-type size distributions as well as for time-dependent distributions arising from coagulation calculations in Sect. 3. Comparing the derived surface brightnesses we propose possible scenarios to account for the coreshine in Sect. 4

\section{Determination of the coreshine surface brightness}

L1506C has been observed as part of the Taurus Spitzer Survey (PI Padgett; Rebull et al. 2010). Two observations per pointing were performed across L1506C resulting in an average integration time per pixel of $25.6 \mathrm{~s}$. Point sources have been identified and masked using the sextractor software (Bertin \& Arnouts 1996). Saturated sources were not identified as point sources and are left in the images. The image has been smoothed by a Gaussian kernel with a width of 2 pixels $\left(2.4^{\prime \prime}\right)$.

As visible in Pagani et al. (2010b), Fig. 1, for many cores, coreshine traces well the extinction pattern visible in the $8 \mu \mathrm{m}$ Spitzer image. This helps to identify if the scattered light indeed comes from the central core part. But for L1506C, no extinction pattern is visible above noise at $8 \mu \mathrm{m}$.

However, L1506C has been observed at $1.2 \mathrm{~mm}$ with MAMBO II with a beam size of $11^{\prime \prime}$ (Pea10). Figure 1 shows the IRAC band 1 image together with the MAMBO II map as contours. The coreshine is remarkably well-correlated with the thermal emission of the central core.

The coreshine surface brightness has been measured over a $10^{\prime \prime}$ square region at the peak of the emission associated with the core. The background level has been estimated for a similar size region to the west of the core in a region with no apparent point sources. The surface brightness including background was determined as $0.25 \pm 0.02 \mathrm{MJy} / \mathrm{sr}$, whereas the background level is $0.21 \pm 0.01 \mathrm{MJy} / \mathrm{sr}$, leading to a coreshine surface brightness above background of $0.04 \pm 0.03 \mathrm{MJy} / \mathrm{sr}$.

We also examined the cold Spitzer L1506C data in the IRAC $4.5,5.8$, and $8.0 \mu \mathrm{m}$ bands to derive additional constraints. All three bands do not show significant enhancement

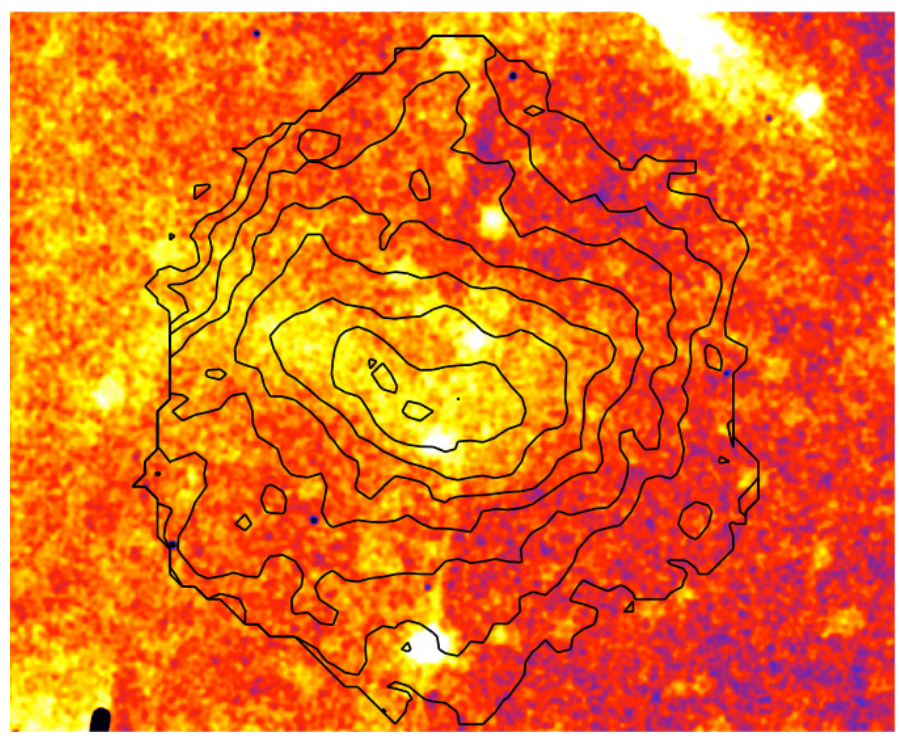

Fig. 1. Core L1506C seen in coreshine and thermal dust emission. Image: Spitzer IRAC channel 1 map of L1506C. North is up and east is to the left. Non-saturated point sources have been identified and masked using sextractor. Contours: MAMBO $1.2 \mathrm{~mm}$ observations (Pea10), in steps of $0.5 \mathrm{mJy} /$ beam up to $5.5 \mathrm{mJy} / \mathrm{beam}$. The field of view is $20^{\prime} \times 13^{\prime}$

or depression of the surface brightness within the noise. For the $8 \mu \mathrm{m}$ band, no background could be derived from the DIRBE maps due to the strong difference in the band position and width. Therefore, we have used an upper limit of $0.03 \mathrm{MJy} / \mathrm{sr}$ (0.3 MJy/sr) for the 4.5 (5.8) $\mu \mathrm{m}$ surface brightness, respectively.

\section{Coreshine from a model core with various grain distributions}

Determining the scattered light surface brightness of a core requires to perform radiative transfer calculations of the usually asymmetric core density structure taking into account the dust opacities of the various grain species as well as the impacting anisotropic radiation field. These quantities have substantial uncertainty ranges, and radiative transfer is too time-consuming to run a grid in the large parameter space with some ten parameters. The only 3D modeling of coreshine so far was performed in Steinacker et al. (2010) for the core L183 for grains increasing in size with gas density. The resulting coreshine images showed stronger gradients than observed and the smooth observational coreshine appearance of L183 and many other cores hints towards a grain distribution with small spatial gradients and/or an efficient mixing of grains. In this analysis, we therefore restrict ourselves to a simple core density model and to determine if proposed size distributions can account to at least reach the coreshine surface brightness level observed in L1506C, without any attempt to model the complex shape of the core, and refer to future studies concerning a full 3D modeling. Moreover, we will neglect spatial variations in the grain size distribution with reference to the results from L183 that such variations cause stronger gradients in the coreshine appearance than observed.

We use the spherically symmetric density distribution proposed for L1506C in Pea10, with an outer radius of $R_{\mathrm{c}}=$ $3 \times 10^{4}$ au, a maximal density of $5 \times 10^{10} \mathrm{~m}^{-3}$, and a kink radius of $R_{\mathrm{c}}=10^{4}$ au where the flat inner profile turns into a powerlaw. The impacting radiation field is approximated by the Zodiacal 


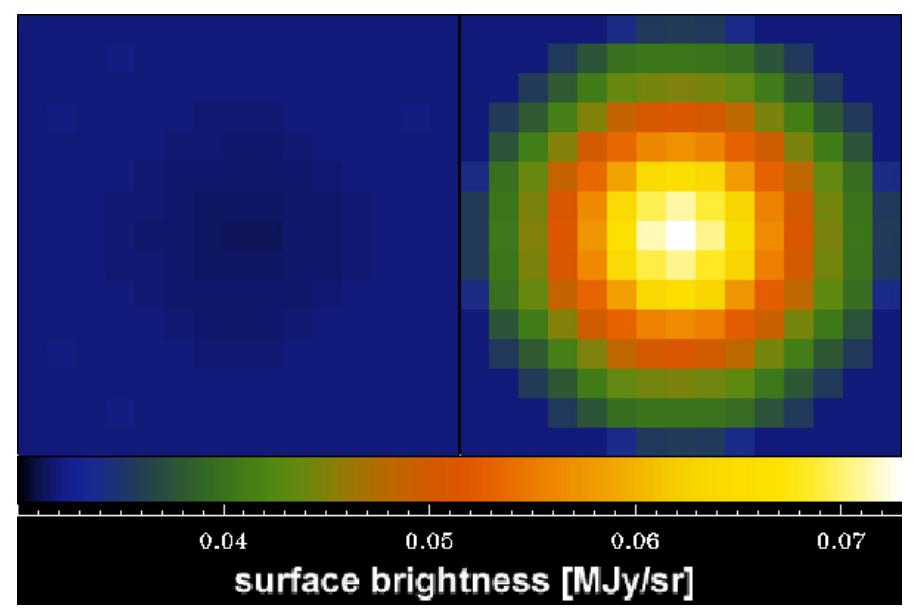

Fig. 2. Scattered light images of a model core with overall properties like L1506C using an MRN size distributions extending to grain sizes of $0.25 \mu \mathrm{m}$ (left) and $0.65 \mu \mathrm{m}$ (right). The total dust mass is kept constant. The background surface brightness is $0.032 \mathrm{MJy} / \mathrm{sr}$ and the coreshine surface brightness above background is $0.04 \mathrm{MJy} / \mathrm{sr}$ for L1506C.

light-subtracted $3.5 \mu \mathrm{m}$ DIRBE map provided by LAMBDA ${ }^{1}$ transformed to the core location at the Galactic coordinates $(l, b)=\left(171.1^{\circ},-17.57^{\circ}\right)$. The background radiation along the line-of-sight was derived from the DIRBE map since the absolute background is provided as opposed to the Spitzer observations. Due to the large beam of DIRBE the surface brightness in each pixel is contaminated by point sources and we subtracted this surface brightness using the WISE point source catalogue leading to a value of $0.032 \pm 0.02 \mathrm{MJy} / \mathrm{sr}$ for the background.

\subsection{Models based on pristine MRN distributions}

The first scenario we discuss is to mix the core gas with a stationary size distribution commonly found in the diffuse interstellar medium (ISM), with a powerlaw distribution as described in Mathis et al. (1977) (abbreviating the distribution MRN) and grain radii up to $0.25 \mu \mathrm{m}$. We use a version of the radiative transfer code applied in Steinacker et al. (2010) that is taking advantage of the assumption of no spatial gradients in the size distribution (for details, see Sect. 2.4 in Steinacker et al. 2013). Dust opacities were taken from Draine \& Lee (1984) and Draine (2003). As shown by Ormel et al. (2011), considering more realistic porous grains will decrease the opacities at $3.6 \mu \mathrm{m}$. Since the optical depth encountered in the calculations remain below 1 in most cases, considering a filling factor of 1 means deriving an upper limit for the scattered light surface brightness.

Figure 2 shows images of the core at a wavelength of $3.6 \mu \mathrm{m}$ for an MRN grain size distribution. In the left image, the distribution includes grains with a radius up to $0.25 \mu \mathrm{m}$. The core is seen in slight absorption against the background with no visible coreshine. We successively raised the upper size limit while keeping the total dust mass constant with a mass ratio of gasto-dust of 120. The right image was calculated using grains up to $0.65 \mu \mathrm{m}$ in size, reproducing the observed surface brightness of coreshine in L1506C around 0.07 MJy/sr. Within the uncertainties and approximations used in the modeling this means that the coreshine reveals a large grain component being present in all parts of L1506C. Interpreted as a pristine component, it could originate either from large-scale dust production and processing cycles of the ISM before the Taurus region was formed or from

\footnotetext{
http://lambda.gsfc.nasa.gov
}

collisional growth in transient density fluctuations in the filament before L1506C came to being.

\subsection{Models based on grain growth in cores}

Alternatively, we assume that the large grains are formed in the core as a consequence of collisional growth due to the turbulent relative velocities of the grains and the high density. We utilize a time-dependent grain growth model for their distribution which is non-trivial also because the core may change its properties during the evolution. Ossenkopf (1993) considered the growth of fluffy dust agglomerates in dense cores of molecular clouds and found that on timescales below $10^{5} \mathrm{yr}$ the optical properties of the grains change but that no large heavy particles are produced. Weidenschilling \& Ruzmaikina (1994) have studied the collisional evolutions of grains in cores both in static equilibrium and free-fall collapse. They derive powerlaw-shaped size distributions with a cut-off at $1 \mu \mathrm{m}$ for compact grains on a timescale of a few Myr, and $100 \mu \mathrm{m}$ for fluffy grains. Ormel et al. (2009, hereinafter called Oea09) have improved the graingrain collision data by numerical experiments. They assumed that the decay time of the largest eddies with the size of the core is the sound crossing time, and that the fluctuating velocity at the largest scale is given by the sound speed. The turbulence on smaller scales was assumed to follow a Kolmogorov cascade with turn-over times and velocities on the inner scale determined by the Reynolds number (for details see Sect. 2 and Appendix A in Oea09). The turbulence on the core scale was renewed over times larger than one free-fall time. In their model, strongly peaked size distributions develop as long as destructive collisions do not limit the growth of grains, with maximal sizes also around $100 \mu \mathrm{m}$ for a few free-fall times. Here, we apply a modified version of the Oea09 model and have rerun a grid of coagulation calculations for a large sample of physical parameters extending the range compared to Oea09. The grid covered gas number densities from $10^{8}$ to $10^{14} \mathrm{~m}^{-3}$, core radii from 4.8 to $110 \mathrm{kau}$, and allows for fluctuating velocities at the largest scale from 90 to $510 \mathrm{~m} / \mathrm{s}$ instead of restricting to the sound speed. The model includes destructive collisions for high relative velocities, and in fact on longer times the grain size distributions flatten due to the action of destructive impacts as visible in Fig. 3 for $t=10^{7} \mathrm{yr}$. The initial silicate grains were ice-coated spheres and had a radius of $0.05 \mu \mathrm{m}$. The grain size distributions were calculated for evolution times from $10^{4}$ to $10^{7}$ yr. Figures 3 and 4 show example results of calculations with parameters partially resembling the properties of $\mathrm{L} 1506 \mathrm{C}$ with the gas number density $n_{\mathrm{g}}$, the turbulent velocity at the outer radius $v_{L}$ and the core radius $R_{\mathrm{cl}}$. For the low turbulence case in Fig. 3, almost no grain growth is produced after 1 Myr. In the low density case (Fig. 4), grains sizes around $0.7 \mu \mathrm{m}$ were created. Note that the results for a core similar to L1506C have been interpolated from this grid to arrive at the correct number densities.

The Oea09 model does not consider mixing by the same turbulence that is providing the relative velocities and assumes the core to have a single constant density. Since the density in our model core mimicking L $1506 \mathrm{C}$ varies, we constructed a global size distribution by counting for each time step the grains in each cell grown as a function of the local density. This still overestimates the growth at the highest densities as the mixing will at all times remove grains from the region towards lower density ones where growth is less efficient. The resulting size distribution per size interval is shown in Fig. 5 for a growth time of 1 Myr and fluctuating velocities of $300 \mathrm{~m} / \mathrm{s}$. 


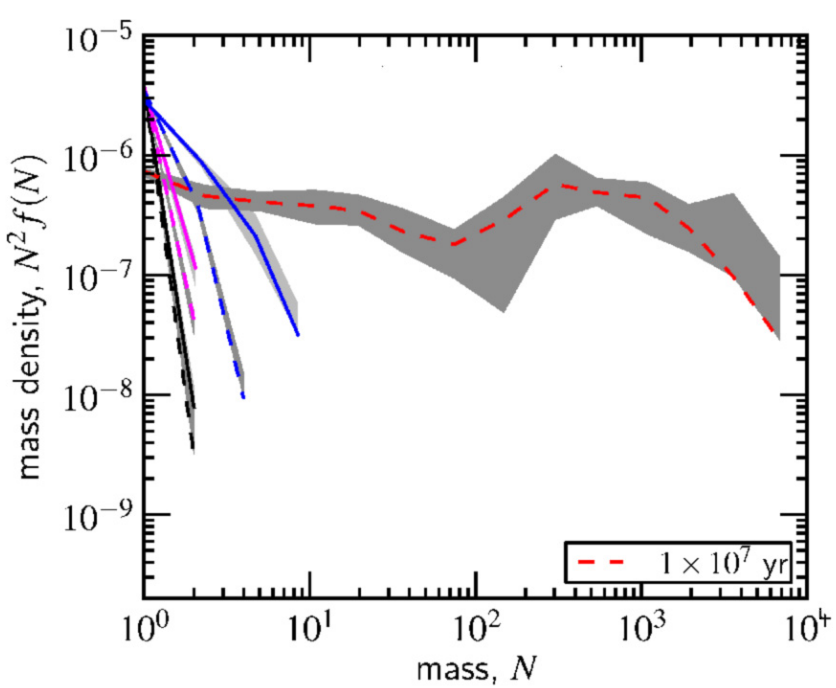

Fig. 3. Mass of the size distribution per logarithmic interval as a function of the grain mass in units of number of initial grains. The curves are at $10^{4}, 3 \times 10^{4}, 10^{5}, 3 \times 10^{5}, 10^{6}, 3 \times 10^{6}, 10^{7} \mathrm{yr}$ continuously increasing their mass center. The gas density is $n=1.3 \times 10^{11} \mathrm{~m}^{-3}$, the turbulent speed $v_{L}=90 \mathrm{~m} / \mathrm{s}$, and the core radius is $R_{\mathrm{c}}=22.7 \mathrm{kau}$. The gray shading denotes the spread in 10 runs. The initial monomers have a size of $0.05 \mu \mathrm{m}$. Only data up to $1 \mathrm{Myr}$ are used in this paper.

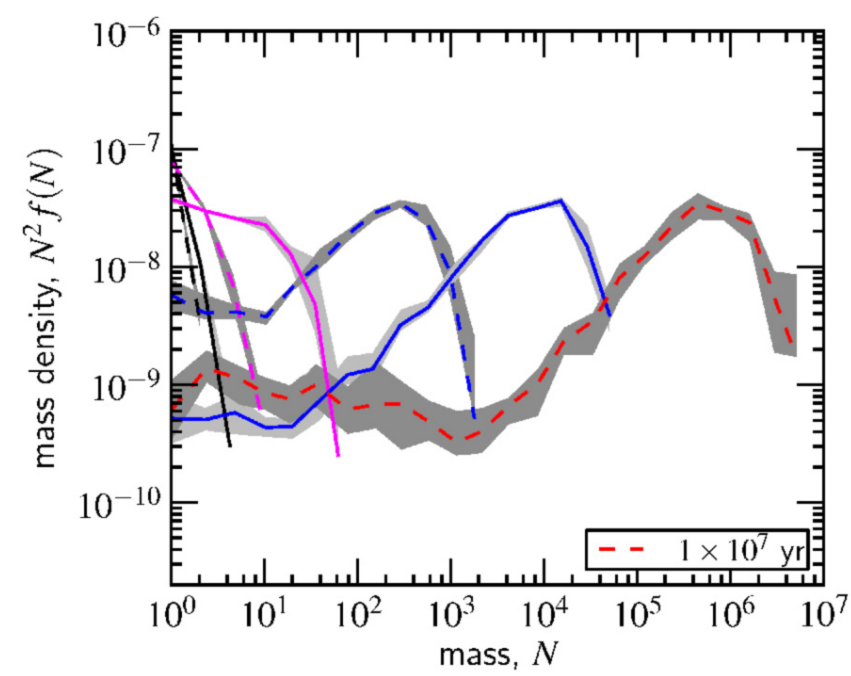

Fig. 4. Same as Fig. 3 with a different set of parameters (low density, mean turbulent velocity): the gas density is $n=3.8 \times 10^{9} \mathrm{~m}^{-3}$, the turbulent speed $v_{L}=300 \mathrm{~m} / \mathrm{s}$, and the core radius is $R_{\mathrm{c}}=22.7 \mathrm{kau}$.

We use these various size distributions to explore which of them would create enough scattered light to explain the observed coreshine of L1506C. We start with the currently observed turbulence and density properties. While the free-fall time scale is of the order $10^{5}$ years, thermal pressure and magnetic fields are discussed to extend the lifetime of pre-stellar cores from several to many free-fall times (see time scale discussion in Oea09). Since we want to explore the conditions to produce measurable coreshine, we consider that the core density was at the value we observe today for at $1 \mathrm{Myr}$ in order to enable efficient grain growth. Assuming fluctuating velocities to be $90 \mathrm{~m} / \mathrm{s}$ near the observed value for the turbulent eddy with the size of the core, maintaining the turbulence at the largest level for $1 \mathrm{Myr}$, the calculation leads to no measurable coreshine surface brightness in $1 \mathrm{Myr}$ (Fig. 6, left). This simple calculation suggests that if L1506C

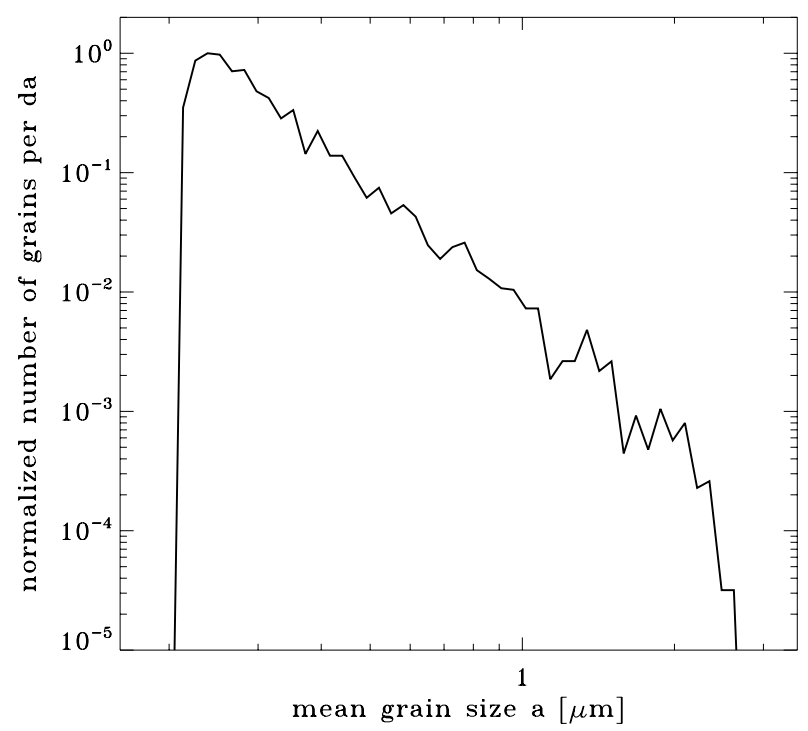

Fig. 5. Normalized size distribution per size interval as a function of the mean grain size. The distribution was obtained allowing growth at all cells according to the local density following the Oea09 calculations and finally mixing all grains to a constant size distribution.

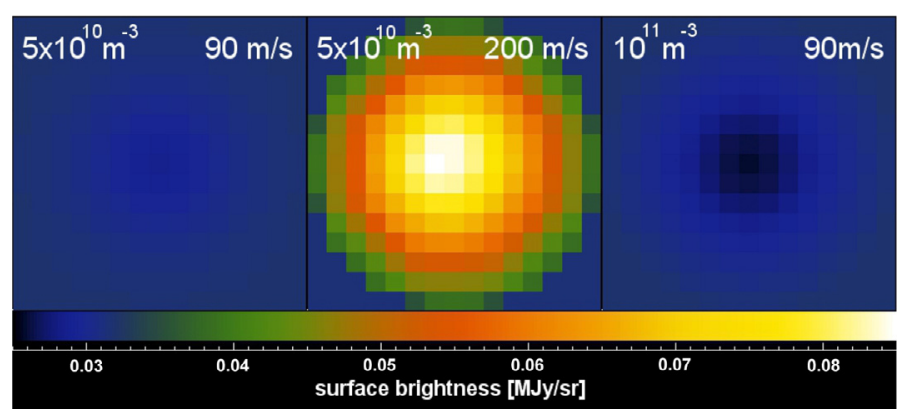

Fig. 6. Scattered light images of a model core like L1506C assuming a growth time of $10^{6} \mathrm{yr}$. In each image, the peak core density is given left, the right number is the turbulent speed in the largest eddy.

would have had the density distribution and the turbulence level that we see today with only $0.05 \mu \mathrm{m}$-sized grains at the onset of growth, applying even the most optimistic growth model yields that L1506C should not harbor larger grains nor should it show coreshine.

We performed radiative transfer calculations using the same set-up but a stronger level of turbulence than currently observed. We chose the outer eddy to have fluctuation velocities of $200 \mathrm{~m} / \mathrm{s}$ comparable to the value derived from ${ }^{13} \mathrm{CO}$ linewidth measurements of about $400 \mathrm{~m} / \mathrm{s}$. Velocities on smaller scales are smaller according to the assumed Kolmogorov cascade.

In the corresponding image (Fig. 6 middle), coreshine is seen at the observed surface brightness level. But mixing was only performed after $10^{6} \mathrm{yr}$ instead of continuous mixing. To estimate the impact of this approximation on the largest grains, we consider a grain that starts in the densest region. Given the centrally peaked gas density distribution, the grain will remain in regions with a central density only about $1 / 3\left(r_{0} / R_{\mathrm{c}}\right)$ of the turnover time. As coreshine is dominated by the largest grains (see Steinacker et al. 2010), a more realistic mixing will result in a coreshine surface brightness too low to account for the observed value. Increasing the turbulent velocity even more is possible, but only up to the sound speed since velocities in pre-stellar low-mass cores remain subsonic. Moreover, the model calculations will 
also overestimate the growth since they assume a Kolmogorov turbulence decay while the linewidth measurements indicate a lack of turbulence in the core.

Aside of considering a turbulence that has been stronger in the past, we can also assume that the core has been denser due to the action of large-scale motions which are tearing the core apart. This would follow the general picture of turbulent star formation and the higher density would let grains grow more efficiently. The right image in Fig. 6 shows the core assuming the turbulence level observed today but with a density being a factor 2 larger. Grain growth is still insufficient to arrive at grains large enough to produce the right coreshine level. Finally we have chosen $300 \mathrm{~m} / \mathrm{s}$ as turbulent speed of the largest eddy and an increased density by shrinking the core and assuming the same mass. We were able to produce coreshine well in excess of the observed value.

We also determined the $4.5 \mu \mathrm{m}$ surface brightness for all models described here. The peak values never exceeded the upper limit of $0.03 \mathrm{MJy} / \mathrm{sr}$ derived from the data. The only exception is the model with both increased turbulent speed and density, and in this case the measured $3.6 \mu \mathrm{m}$ peak values is a tighter constraint. In a further publication we will discuss the more recent and more sensitive warm Spitzer measurements for this core.

\section{Conclusions}

We have investigated the scattered light observed from the central part of L1506C in the Spitzer channel 1 and put forward two scenarios to account for the coreshine surface brightness. Mixing the core case with a constant MRN dust size distribution using a size limit of $0.25 \mu \mathrm{m}$ does not lead to sufficient scattered light to explain the observed surface brightness of cores with coreshine.

The core may host a component of large grains with sizes up to $0.65 \mu \mathrm{m}$ that has not been formed in the core rather than being a primitive component from earlier star formation cycles before the Taurus filaments came to being. Using a simple density structure, an ISRF based on the DIRBE map plus a local component from the star formation region, and standard dust opacities we were able to reproduce the coreshine level with our radiative transfer calculations.

An implication of the existence of a global primitive largegrain component would be that most low-mass cores comparable to L1506C should show coreshine as far as the background level allows its detection, shielding effects do not block the radiation either during illumination or on its way to the observer, or large-scale processes like supernovae have modified the size distribution (Andersen et al. 2011; Pagani et al. 2012). A future publication will investigate this prospective.

Another possibility is that the large grains responsible for the scattering have been formed in the core. To enable efficient grain growth, we maintained the turbulence on the largest scale for $1 \mathrm{Myr}$ which is a basic assumption of the gravo-turbulent scenario for star formation. But even with the most optimistic grain growth model, and increasing either the turbulence or the density beyond the currently observed values, we failed to reproduce the observed coreshine within the limits of our simple model. However, our results indicate that grain growth in a core that is denser and more turbulent could lead to the observed coreshine surface brightness. Within the gravo-turbulent scenario, the same large-scale motions that would have created the density maximum in the filament at the location of L1506C could have torn the core partially apart leaving just the gravitationally bound part. The question is if the velocity gradients that have been inferred from the emission line analysis along a cut in Pea10 are in agreement with this scenario. Since turbulence on the largest scales decays slowest in the Kolmogorov picture, it is on the large-scales that remnants of such a tearing motion should still be seen. The observed outward motion of the envelope at large scales can be interpreted in this way.

It is more complex to access the present kinematical signatures on the smaller scales, that is on the scale of the core. First, the turbulence on smaller scales decays faster and is re-created by turbulent cascading with new kinematical signature. This leaves no trace of the history of the fragment on small scales. Second, the kinematic structure of the core may also be affected by self-gravity: the change in kinematical properties appears to happen right at a Jeans length of about $3 \times 10^{4}$ au which is also the core radius. Furthermore, the action of magnetic fields and density gradients can alter the behavior of the turbulence. But the fact that today only a low level of turbulence is observed suppressing efficient grain growth hints towards an evolution which has seen stronger turbulence at former times.

We have not made use of the other interesting peculiarity of the core: its strong depletion. Chemical model calculations for transient density fluctuations forming core-like density enhancements have been performed (Garrod et al. 2005). Under the assumption of a Gaussian core density profile whose amplitude varies as a time-dependent Gaussian, they probed the chemical evolution at various times and points in the core with a 221 species chemical code. It was found that the freeze-out in the high-density regions maintains for some time after the strongest compression has passed until the extinction drops below a critical value and the molecules are evaporated back into the gas phase. This result would be consistent with finding a core in the process of decreasing density and yet showing substantial depletion.

While running a chemistry model for a core with the properties of L1506C and a given evolutionary sequence for the density structure is possible, comparison with the line data would also require to run a line transfer model. The parameter space to be covered will need careful exploration and this advanced effort is beyond the scope of this paper, but an interesting perspective for future work on this remarkable core.

The presented results and both scenarios are in agreement with L1506C being a pre-stellar core in the making as proposed by Pea10: despite its turbulent and dense history the low-density core appears to be gravitational bound and showing infall motions. But within the picture of grain growth in cores, the presence of coreshine and depletion might indicate that it is in the re-making.

Acknowledgements. We thank David Flower and Wing-Fai Thi for valuable comments, Laurent Pagani for comments and providing the MAMBO map, and the referee for suggestions helping to improve the paper. J.S. and M.A. acknowledge support from the ANR (SEED ANR-11-CHEX-0007-01). CWO acknowledges support for this work by NASA through Hubble Fellowship grant No. HST-HF-51294.01-A awarded by the Space Telescope Science Institute, which is operated by the Association of Universities for Research in Astronomy, Inc., for NASA, under contract NAS 5-26555. This work is based on observations made with the Spitzer Space Telescope, which is operated by the Jet Propulsion Laboratory, California Institute of Technology under a contract with NASA.

\section{References}

Andersen, M., Rho, J., Reach, W. T., Hewitt, J. W., \& Bernard, J. P. 2011, ApJ, 742,7

Bergin, E. A., \& Tafalla, M. 2007, ARA\&A, 45, 339

Bertin, E., \& Arnouts, S. 1996, A\&AS, 117, 393 
Draine, B. T. 2003, ApJ, 598, 1017

Draine, B. T., \& Lee, H. M. 1984, ApJ, 285, 89

Pagani, L., Steinacker, J., Bacmann, A., Stutz, A., \& Henning, T. 2010b, Science, 329, 1622

Garrod, R. T., Williams, D. A., Hartquist, T. W., Rawlings, J. M. C., \& Viti, S. 2005, MNRAS, 356, 654

Keto, E., \& Caselli, P. 2008, ApJ, 683, 238

Mac Low, M.-M., \& Klessen, R. S. 2004, Rev. Mod. Phys., 76, 125

Mathis, J. S., Rumpl, W., \& Nordsieck, K. H. 1977, ApJ, 217, 425

Ormel, C. W., Paszun, D., Dominik, C., \& Tielens, A. G. G. M. 2009, A\&A, 502, 845

Ormel, C. W., Min, M., Tielens, A. G. G. M., Dominik, C., \& Paszun, D. 2011, A\&A, 532, A43

Ossenkopf, V. 1993, A\&A, 280, 617

Pagani, L., Ristorcelli, I., Boudet, N., et al. 2010a, A\&A, 512, A3
Pagani, L., Lefèvre, C., Bacmann, A., \& Steinacker, J. 2012, A\&A, 541, A154

Pagani, L., Lesaffre, P., Jorfi, M., et al. 2013, A\&A, 551, A38

Qian, L., Li, D., \& Goldsmith, P. 2012, ApJ, 760, 147

Rebull, L. M., Padgett, D. L., McCabe, C.-E., et al. 2010, ApJS, 186, 259

Steinacker, J., Pagani, L., Bacmann, A., \& Guieu, S. 2010, A\&A, 511, A9

Steinacker, J., Baes, M., \& Gordon, K. 2013, ARA\&A, 51, 63

Vázquez-Semadeni, E., Ballesteros-Paredes, J., \& Klessen, R. S. 2003, ApJ, 585, L131

Weidenschilling, S. J., \& Ruzmaikina, T. V. 1994, ApJ, 430, 713

Ysard, N., Abergel, A., Ristorcelli, I., et al. 2013, A\&A, 559, A133 